SAT0108

RESPONSE TO ABATACEPT IS ASSOCIATED WITH THE INHIBITION OF PROTEASOME B1I EXPRESSION INT CELLS OF PATIENTS WITH RHEUMATOID ARTHRITIS

K. Ghannam ${ }^{1}$, L. Martinez Gamboa ${ }^{1}$, C. Kedor ${ }^{1}$, L. Spengler ${ }^{2}$, G.

R. Burmester ${ }^{1}$, E. Feist ${ }^{3} .{ }^{1}$ Charité - Universitätsmedizin Berlin, Department of Rheumatology and Clinical Immunology, Berlin, Germany; ${ }^{2}$ Dr. med. Lydia Spengler, Innere Medizin und Rheumatologie, Radebeul, Germany; ${ }^{3}$ Specialist Hospital Vogelsang Gommern GmbH, Klinik für Rheumatologie, Gommern, Germany

Background: Abatacept (CTLA4-Ig) is a biological DMARD (bDMARD) for treatment of rheumatoid arthritis (RA) and selectively modulates the co-stimulatory signal by CD28: CD80/CD86 interaction required for T cell activation. CD28 mediated signaling regulates many $T$ cell functions including cytokine production. The role of proteasome was approved in many autoimmune diseases, and the effect of Abatacept on modifying cytokines including interferon- $\gamma$ that alters proteasome proteolytic activities was shown in RA.

Objectives: To characterize the effect of Abatacept on the expression and proteolytic activity of the immunoproteasome.

Methods: Effects of Abatacept on the proteasome system were investigated in 39 patients with rheumatoid arthritis over a period of 24 weeks. Using real time PCR, transcript expression levels of constitutive and corresponding immunoproteasome catalytic subunits were investigated at baseline (T0), week 16 (T16) and week 24 (T24) in sorted blood cells. Proteasomal activity and induction of apoptosis after proteasome inhibition were also evaluated in cellular subsets.

Results: Upon treatment with Abatacept, remission or low disease activity according to DAS28 was achieved in $55 \%$ of patients at T16 and in $70 \%$ at T24. By Two- (time and type of response) way ANOVA, a significant reduction of proteasome immunosubunit $\beta 1 \mathrm{i}$ expression was shown only in CD4+ and CD8+T cells of prolonged responders at both T16 and T24 ( $P=0.0390$ and $P=0.0198$, respectively). One-way ANOVA analysis for each response group separately confirmed the results and showed significant reduction at T24; $P=0.0396$ difference between T0 and T24, $P=0.0260$ between T16 and T24 in CD4+ T cells of the same group. Abatacept did not influence chymotrypsin like activity of proteasome, which is carried out by the subunit $\beta 5 i$ and had no effect on induction of apoptosis under exposure to a proteasome inhibitor in-vitro.

Conclusion: Treatment with Abatacept showed a clear effect on the expression of the proteasome immunosubunit $\beta 1$ i. This phenomenon was only seen in CD4+ and CD8+ T cells of prolonged responding patients with RA suggesting an association between persistent induction of $\beta 1 \mathrm{i}$ and failure to the T cell directed therapy with Abatacept. References:

[1] Keating, G.M., Abatacept: A Review of its Use in the Management of Rheumatoid Arthritis. Drugs, 2013. 73(10): p. 1095-1119.

[2] Ferrington, D.A. and D.S. Gregerson, Immunoproteasomes: Structure, Function, and Antigen Presentation. Progress in molecular biology and translational science, 2012. 109: p. 75-112.

[3] Marti, L., et al., Alterations in Cytokine Profile and Dendritic Cells Subsets in Peripheral Blood of Rheumatoid Arthritis Patients before and after Biologic Therapy. Annals of the New York Academy of Sciences, 2009. 1173: p. 334-42. Disclosure of Interests: Khetam Ghannam: None declared, Lorena Martinez Gamboa: None declared, Claudia Kedor Consultant of: Advisory Board for Novartis Pharma GmbH, Lydia Spengler: None declared, Gerd Rüdiger Burmester Consultant of: AbbVie Inc, Eli Lilly, Gilead, Janssen, Merck, Roche, Pfizer, and UCB Pharma, Speakers bureau: AbbVie Inc, Eli Lilly, Gilead, Janssen, Merck, Roche, Pfizer, and UCB Pharma, Eugen Feist Consultant of: Novartis, Roche, Sobi, Lilly, Pfizer, Abbvie, BMS, MSD, Sanofi, Speakers bureau: Novartis, Roche, Sobi, Lilly, Pfizer, Abbvie, BMS, MSD, Sanofi

DOI: 10.1136/annrheumdis-2020-eular.4265

\section{SAT0109 \\ DISTINCT EFFECTS OF METHOTREXATE IN COMBINATION WITH ADALIMUMAB OR ETANERCEPT ACCORDING TO CCP ANTIBODY STATUS IN PATIENTS WITH RHEUMATOID ARTHRITIS}

M. Greenwood ${ }^{1}$, M. Shipa ${ }^{1}$, M. Ehrenstein ${ }^{1} .{ }^{1}$ University College London, London, United Kingdom

Background: Considerable evidence suggesting enhanced efficacy of anti-TNF therapy for rheumatoid arthritis (RA) when co-administered with methotrexate (MTX) led us to explore the applicability of the published data to our patient cohort. Objectives: This retrospective study aimed to investigate the efficacy of MTX in combination with adalimumab (ADA) and etanercept (ETN) for RA, examining changes in disease activity score (DAS28-CRP) on first starting treatment, and rates of withdrawal due to inefficacy over up to 10 years of treatment in routine clinical practice.

Methods: 309 patients with RA who had started ADA or ETN as a first-line biologic at UCLH from 2003 onwards were identified. Demographic and treatment details, DAS28-CRP (DAS) at the biologic-naïve baseline and at a first appointment for review on anti-TNF were extracted from clinical records. ANOVA was used to investigate the level of association between change in DAS and anti-TNF type, concomitant therapy, anti-CCP (CCP), rheumatoid factor (RF), age, disease duration and number of DMARD trials by anti-TNF initiation, year of initiation and gender. Kaplan-Meier with log rank tests were used to explore associations between anti-TNF type, MTX, CCP and RF and rates of withdrawal due to inefficacy, and Cox Proportional Hazards to check whether these remained significant after controlling for any other significant factors.

Results: 179 patients were on ADA and $130 \mathrm{ETN} ; 44 \%$ were on concomitant MTX (Table 1).

Table 1. Numbers of patients co-prescribed MTX with ADA or ETN according to CCP status

\begin{tabular}{lcc}
\hline & CCP positive $\mathrm{n}$ & Total $\mathrm{n}$ \\
\hline ETN without MTX & 37 & 54 \\
with MTX & 57 & 76 \\
ADA without MTX & 54 & 82 \\
with MTX & 65 & 97 \\
\hline
\end{tabular}

Median follow up since anti-TNF initiation was 7 years. Median age at initiation was 51 years, and disease duration 4 years. $82 \%$ were female. 241 were seropositive, including 24 positive for CCP only and 28 RF only.

Mean biologic-naïve (baseline) DAS was 5.75, and 3.52 on first review (mean 20 weeks of treatment, $n=274$ ) with mean change -2.23 . MTX, even accounting for dose, failed to explain a significant amount of the variance in DAS response at first review either as a main effect o in interaction with anti-TNF type, CCP or RF. The only significant factor was 4+ DMARD trials prior to initiation (lower mean DAS response, $\mathrm{p}=0.010$ ).

Next, we analysed withdrawal of anti-TNF for inefficacy over up to 10 years. Kaplan-Meier analyses revealed that CCP positive patients had higher inefficacy withdrawal rates $(p=0.004)$. $O$ note, CCP positive patients treated with MTX had lower withdrawal rates for inefficacy (Figure 1. $\mathrm{p}=0.006$ ). Intriguingly, there was a trend towards higher rates in patients treated with MTX if CCP negative $(p=0.14)$. Co-administration of MTX appeared to reduce rates of inefficacy withdrawal for patients on ETN ( $p=0.006)$ but not ADA $(p=0.784)$. For CCP positive patients on ETN, MTX was strongly associated with lower rates of inefficacy withdrawal (Figure 1, $p=.002$ ). CCP status and the $\mathrm{CCP}^{*} \mathrm{MTX}$ interaction remained significant in the multivariate Cox mode (Table 2). The MTX ${ }^{\star} E T N$ interaction was also included by the backward stepwise variable selection procedure although with $\mathrm{p} 0.069$.

Table 2. Variables in the Cox Model

\begin{tabular}{lcc}
\hline & $\begin{array}{c}\text { Hazard Ratio for discontinuation related to } \\
\text { inefficacy }\end{array}$ & $\begin{array}{c}95 \% \mathrm{Cl} \\
\text { for } \mathrm{HR}\end{array}$ \\
\hline MTX & 2.18 & $0.93,5.13$ \\
CCP & 3.71 & $1.74,7.92$ \\
CCP*MTX & 0.35 & $0.14,0.91$ \\
ETN*MTX & 0.61 & $0.36,1.04$ \\
Disease duration > 5 years & 0.61 & $0.41,0.90$ \\
Gender (male) & 0.62 & $0.36,1.05$ \\
4+ DMARD trials & 1.65 & $1.02,2.67$ \\
Biologic-naive Global VAS/cm & 1.26 & $1.11,1.42$ \\
\hline
\end{tabular}

Overall model $\mathrm{p}<.0005$

RF with a univariate Cox significance of 0.172 was less closely associated with rate of withdrawal for inefficacy compared to anti-CCP positivity ( $\mathrm{p}$ 0.005).

Conclusion: There was no apparent benefit of MTX with anti-TNF with respect to initial DAS response, but MTX was associated with a reduction in withdrawals for inefficacy though only in CCP positive patients treated with ETN.

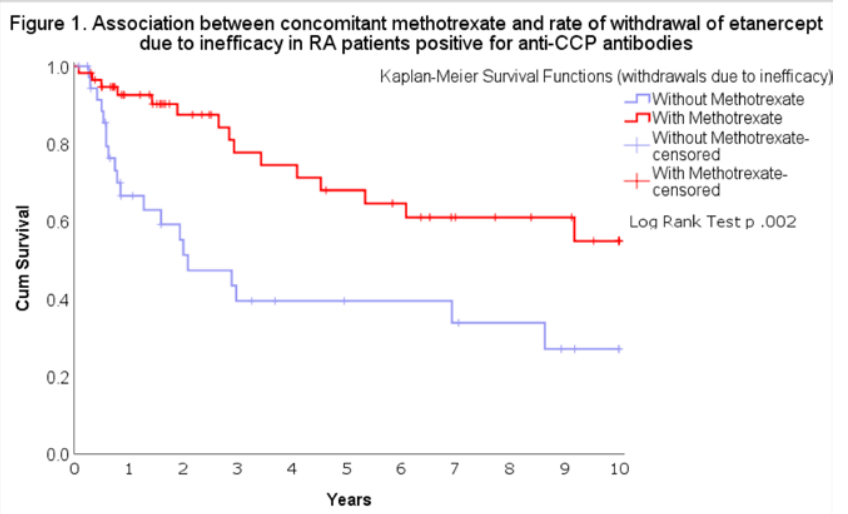

Disclosure of Interests: None declared DOI: 10.1136/annrheumdis-2020-eular.1858 